\title{
Diseño de un material didáctico computarizado para la enseñanza de Oscilaciones y Ondas, a partir del estilo de aprendizaje de los estudiantes
}

\section{Design of a computerized educational material for teaching of oscillations and waves, from students learning style}

Edelmira Ruiz-Macías'
Julio Enrique Duarte

Recibido: septiembre 19 de 2017 Aceptado: diciembre 09 de 2017

\section{Resumen}

En este artículo se presenta el diseño de un material educativo computarizado para la enseñanza de oscilaciones y ondas, orientado a estudiantes de educación media. El proyecto se adelantó con 27 estudiantes del grado undécimo, pertenecientes a la Institución Educativa Técnica Valentín García, de Labranzagrande, Boyacá. La herramienta se diseñó teniendo en cuenta las características sociodemográficas de la población, el acceso a la tecnología y la habilidad para manejarla; igualmente, se consideraron los estilos de aprendizaje de los estudiantes, insumo fundamental para desarrollar un material adecuado al contexto. El diseño se basa en la metodología propuesta por Galvis Panqueva para el desarrollo de software, obteniendo como resultado una herramienta multimodal, en la cual se presentan diferentes actividades que potencializan los estilos de aprendizaje. El material propuesto incluye un menú principal con seis temas, los cuales incorporan actividades e información relacionada con el área de ciencias naturales, haciendo énfasis en la temática de oscilaciones y ondas.

Palabras Clave: aprendizaje significativo, estilos de aprendizaje, materiales educativos computarizados, oscilaciones y ondas.

\begin{abstract}
This paper shows the design of a computerized educational material for the teaching of oscillations and waves, aimed to high school students. It is carried out with 27 students of eleventh grade of the Institucion Técnica Educativa Valentin Garcia de Labranzagrande, Boyacá. The technological tool was designed having in mind the socio-demographic characteristics of the population, access to technology and the ability to use it. Furthermore, students learning styles were considered as a basic input to develop an appropriate material to their context. The design is based on the methodology proposed by Galvis Panqueva for the development of software, getting as a result a multimodal tool, in which different activities that focus on learning styles are presented. The proposed material includes a main menu with six themes, which incorporate activities and information related to the natural sciences subject, emphasizing on oscillations and waves topics.
\end{abstract}

Keywords: meaningful learning, learning styles, computerized educational materials, oscillations and waves.

1 Ingeniera Industrial, Estudiante maestría en TIC aplicadas a las ciencias de la educación, Institución Educativa Técnica Valentín García, Labranzagrande, Colombia. E-mail: edelruiz65@ gmail.com

2 Licenciado en Física, doctor en Ciencias Físicas, Universidad Pedagógica y Tecnológica de Colombia, Duitama, Colombia. E-mail: julioenriqued1@gmail.com 


\section{Introducción}

El gran desarrollo de la tecnología informática y el avance en las comunicaciones, ha provocado un cambio radical en la forma como se percibe la vida actual en todos los ámbitos de la actividad humana (Núñez-Pérez, 2015; Novoa-Ruiz, 2013). En este sentido, las Tecnologías de la Información y Comunicación, TIC, han impactado a las instituciones educativas en los procesos tanto administrativos como académicos (García-Amaya, Fernández-Morales \& Duarte, 2017). Los cambios en la educación implican nuevas formas de relación entre los actores del proceso formativo, a la vez que se brindan nuevas alternativas para el acceso a la información (Avella-lbáñez, SandovalValero \& Montañez-Torres, 2017). Las TIC exigen la adquisición de nuevas competencias, tanto en docentes como en estudiantes, para lograr aprendizajes significativos que conlleven al éxito escolar y prepararlos para su futura vida universitaria y laboral (Niño-Vega, Martínez-Díaz \& Fernández-Morales, 2016).

La física es una ciencia que explica los fenómenos de la naturaleza y su aplicación facilita la creación de tecnología, razón por la cual se fomenta su estudio en todos los niveles educativos (ReyesCaballero, Fernández-Morales \& Duarte, 2016; Duarte, Reyes-Caballero \& Fernández-Morales, 2013). Las investigaciones sobre enseñanza de las ciencias naturales han permitido detectar falencias en la formación conceptual en áreas como: química, biología y física, esto debido a que el estudiante no conecta la teoría vista en la clase tradicional con la vida cotidiana (RodríguezCepeda, 2016; Parra-León, Duarte \& FernándezMorales, 2014).

La actual generación de estudiantes se considera como de Nativos Digitales, ya que nacieron en la era digital y son usuarios permanentes de las tecnologías, con una habilidad consumada para la manipulación de artefactos (García-Llorente, 2015). Su característica principal es sin duda la tecnofilia, pues sienten atracción por todo lo relacionado con las nuevas tecnologías; adicionalmente, con las TIC satisfacen sus necesidades de entretenimiento, diversión, comunicación, información $y$, tal vez, también de formación (Cabero-Almenara, 2015; Buitrago-Guzmán, 2014). En contraste, los docentes, Inmigrantes Digitales, "son aquellos que se han adaptado a la tecnología y hablan su idioma, pero con "un cierto acento". Estos inmigrantes son fruto de un proceso de migración digital que supone un acercamiento hacia un entorno altamente tecnificado, creado por las TIC. Se trata de personas entre 35 y 55 años que no son nativos digitales y han tenido que adaptarse a una sociedad cada vez más tecnificada" (CaberoAlmenara, 2015).

Estas dos generaciones son las que convergen en las aulas actuales, tanto de la educación media como la universitaria, y ven en el uso de las TIC potencialidades enormes para enseñar y aprender de una forma eficiente, motivadora y hasta divertida (Reinón \& Ramos, 2014; JiménezPitre, Vesga \& Martelo, 2017). Sin embargo, la tecnología no soluciona todos los problemas de aprendizaje de los estudiantes, ya que el docente está en la obligación de realizar una planeación adecuada para lograr en su totalidad los objetivos propuestos.

La propuesta aquí presentada consiste en diseñar una herramienta didáctica con la cual los estudiantes relacionen el mundo real con los conocimientos adquiridos en el aula, transformando así su estructura cognitiva, construyendo el concepto claro y duradero del tema de Oscilaciones y Ondas. Esta temática hace parte del plan de área de física del grado undécimo, fundamentado en los estándares básicos del área de ciencias naturales, la cual integra los ejes temáticos de: entorno vivo (biología), entorno químico (química), entorno físico (física), junto con el eje de ciencia, tecnología y sociedad.

El estudio de Oscilaciones y Ondas cobra gran importancia en el mundo de hoy, pues son fenómenos ampliamente utilizados en la 
construcción de instrumentos musicales, en dispositivos para la transmisión y recepción del sonido, en equipos para la transmisión y recepción de radio y televisión, en equipos biomédicos y de aplicaciones industriales, entre otros (PabónFernández, Díaz-Rodríguez \& Pardo-García, 2016; Castro-Galeano, Pinto-Salamanca \& AmayaQuitián, 2014; Mora-Mendoza, Sarmiento-Santos \& Casallas-Caicedo, 2014; Martínez-Ovalle, ReyesCaballero \& González-Puin, 2013).

Estos dispositivos y equipos, de los cuales el hombre moderno depende grandemente, constituyen sistemas en los que tienen lugar procesos oscilatorios y ondulatorios; adicionalmente, tienen como punto de partida las siguientes temáticas: sonido, luz, electricidad y magnetismo (Altamirano-Santillán, VallejoVallejo \& Cruz-Hurtado, 2017; Fernández-Morales, Duarte \& Parra-León, 2014; Fernández-Morales \& Duarte, 2014). Lo anterior implica que el estudio de estos conceptos debe ser claro y duradero, para lograr desempeños destacados en el rendimiento académico y en las pruebas externas, así como en el futuro ejercicio profesional, en especial de quienes se dediquen al estudio de las ciencias básicas y aplicadas (Angarita-Velandia, FernándezMorales \& Duarte, 2016).

La propuesta pedagógica para la enseñanza de Oscilaciones y Ondas, en estudiantes de grado undécimo, contempla un enfoque Cognitivista, utilizando el modelo de aprendizaje significativo. La herramienta didáctica empleada fue un Material Educativo Computarizado, MEC, tipo página web, quecontieneelementos multimedia, interactividad e integración de laboratorios virtuales. En la siguiente sección se presenta el marco teórico y la metodología para el desarrollo de la herramienta. Luego se presentan los resultados, iniciando con el análisis de los estilos de aprendizaje de los estudiantes, insumo fundamental para el diseño. EI MEC contiene elementos multimedia, como videos e imágenes, para estudiantes cuyo estilo de aprendizaje es visual y auditivo. Interactividad con actividades creadas en Educaplay embebidas en la aplicación; simulaciones y laboratorios virtuales para Kinestésicos; así como textos en pdf y presentaciones para los estudiantes con estilo Lector.

\section{Marco teórico y metodología}

\subsection{Fundamentación teórica}

\section{Lineamientos pedagógicos}

La Teoría Cognitiva, en líneas generales, concibe el aprendizaje como un proceso interno que toma en consideración la estructura cognitiva del individuo, la cual es elemento determinante del aprendizaje y de comprensión significativa de los nuevos conocimientos (De León \& Suárez, 2008). El enfoque Cognitivo tiene en cuenta los conocimientos previos del estudiante, ya que es la base para planificar y poner en marcha cualquier estrategia, y así proporcionar herramientas de aprendizaje para que los estudiantes puedan cambiar, o modificar, su estructura conceptual hacia una de tipo científico, evitando la memorización aislada de conceptos o datos y facilitando la comprensión de los mismos (CortésRamos, Artunduaga, Suárez \& Marín-Peña, 2016).

Un aprendizaje, para que se pueda denominar así, debe ser significativo. Existe un grupo de autores conesteenfoque, resaltandolosaportes de Ausubel referentes al Aprendizaje Significativo; este se logra cuando el estudiante puede relacionar los nuevos conceptos con su experiencia individual, no de forma arbitraria, sino organizados en estructuras cognitivas (Bernal-Pinzón, 2017). En algunas ocasiones estos conocimientos hacen referencia a los impartidos en la escuela, pero se deben tener en cuenta los conocimientos de su ambiente y las observaciones diarias del estudiante, para hacer que los relacione y así observar su utilidad (González-Otero, 2017). Entre los objetivos de la educación se encuentran: preparar al estudiante para la vida, enseñarlo a pensar, a que valore la significación del conocimiento y el proceso mismo de aprendizaje, de forma que se estimule 
cada vez más la independencia, creatividad y autorregulación en la obtención de nuevos conocimientos (Murcia \& Henao, 2015).

Un entorno de enseñanza-aprendizaje es el escenario físico donde un estudiante, o comunidad de estudiantes, desarrolla su trabajo, incluyendo todas las herramientas, documentos y otros artefactos que pueden encontrar en dichos escenarios (Angarita-Velandia, Fernández-Morales \& Duarte, 2014). En otras palabras, el entorno de aprendizaje incluye no solo el escenario físico, sino también los materiales, estrategias y aspectos socioculturales relacionados con el contexto donde se adelanta el proceso formativo. En este punto es relevante la estrategia didáctica, entendida como una instancia que acoge tanto métodos, como medios y técnicas, considerando que el concepto proporciona mayor flexibilidad y utilidad en relación al tratamiento de las TIC en el proceso didáctico (Salinas, 2004).

Igualmente importante, para el diseño de un entorno de aprendizaje efectivo, es el estilo de aprendizaje de los estudiantes (González-Calixto, Patarroyo-Durán \& Carreño-Bodensiek, 2017). En este sentido, Fleming y Mills desarrollaron una propuesta para clasificar a las personas de acuerdo a su preferencia en la modalidad sensorial, a la hora de procesar información o contenidos educativos (Fleming, 2001). Los autores consideran que las personas reciben información constantemente a través de los sentidos y que el cerebro selecciona parte de esa información e ignora el resto. Las personas seleccionan la información a la que le prestan atención en función de sus intereses, pero también influye cómo se recibe la información (Velásquez, Ortiz \& Rodríguez, 2016).

Existe un cuestionario denominado instrumento de VARK, acrónimo en inglés de las cuatro letras iniciales de las preferencias modales sensoriales, a saber: Visual, Aural, Read/Write y Kinesthetic (Fleming, 2001). Estas preferencias perceptuales se caracterizan por lo siguiente:
- Visual (visual): preferencia por maneras gráficas y simbólicas de representar la información.

- Auditivo (aural): preferencia por escuchar la información.

- Lectura - escritura (read/write): preferencia por información impresa en forma de palabras.

- Quinestésico (kinesthetic): preferencia perceptual relacionada con el uso de la experiencia y la práctica, ya sea real o simulada.

\section{Tecnologías de la información y la comunicación}

Las Tecnologías de la Información y la Comunicación, TIC, se deben entender como aquellas herramientas basadas en la tecnología digital que involucran el computador y la Internet, permitiendo almacenar, procesar, recuperar, transmitir y presentar cantidades masivas de información (Cabero-Almenara, 2015). Estas tecnologías incluyen las de facilitar y gestionar información software, bases de datos y multimedia, entre otras; igualmente incluyen las nuevas tecnologías alrededor de Internet, como: foros, chat, listas de distribución y plataformas para e-learning (Gómez-Soto, Sánchez-Toro \& Benavides-Salazar, 2017; Jiménez-Pitre, Martelo-Gómez \& Peña-Pertúz, 2017; Tangarife-Chalarca, 2013). Además, se entiende por material digital el software, los programas o aplicaciones utilizadas por los computadores para facilitar y gestionar información (Jaramillo, Castañeda \& Pimienta, 2009; Mercado-Ramos, Zapata \& Ceballos, 2015).

Con las potencialidades que ofrecen las TIC, los ambientes de aprendizaje se han flexibilizado en tiempo y espacio mediante las herramientas de comunicación y colaboración, configurando modalidades de estudio emergentes, donde estos ambientes se transforman en espacios de interacción virtual, denominados Ambientes Virtuales de Aprendizaje, AVA (Parada-Hernández \& 
Suárez-Aguilar, 2014; Ricardo, Borjas, Velásquez, Colmenares \& Serge, 2013).

Estos escenarios educativos, basados en la tecnología como mediadora y favorecedora del aprendizaje, representan una posibilidad para articular y conjugar las diferentes áreas del conocimiento, a fin de desarrollar propuestas desde una dimensión científica y socioafectiva (Pérez \& Telleria, 2012; Cárdenas-Soler \& Martínez-Chaparro, 2015).

\section{Materiales educativos computarizados}

Los Materiales Educativos Computarizados, MEC, son recursos educativos en formato digital que manejan conceptos breves, claros y precisos de lo que se quiere enseñar a través del computador. Para Galvis-Panqueva (1994), MEC es la denominación otorgada a las diferentes aplicaciones informáticas, cuyo objetivo final es apoyar el aprendizaje. Estos materiales se caracterizan porque es el estudiante quien controla el ritmo de aprendizaje, la cantidad de ejercicios, decide cuándo abandonar y reiniciar, pudiendo interactuar reiteradas veces; adicionalmente, el docente encuentra en ellos una ayuda significativa, pues en muchos casos en los MEC se registra toda la actividad del estudiante.

Para la elaboración de un software educativo es necesario tener en cuenta aspectos tanto pedagógicos como técnicos; su desarrollo consiste en una secuencia de pasos que permiten crear un producto adecuado a las necesidades de los estudiantes, necesidades que deben ser rigurosamente analizadas por la persona que elabora el material (Leguizamón-González, 2011).

Los MEC, dependiendo del objetivo que persiguen, del momento educativo en que se vayan a utilizar o de la complejidad en su diseño, pueden presentar diversas tipologías. Existen entonces materiales de tipo algorítmico, de ejercitación y práctica, sistemas tutoriales, heurísticos, juegos educativos, simuladores, micro mundos exploratorios, sistemas expertos y tutores inteligentes, por mencionar algunos (Martínez-Marín \& Cantú-Munguía, 2017; Cerón-Correa, Salazar-Jiménez \& Prieto-Ortíz, 2013; Millán-Rojas, Gallego-Torres \& Chico-Vargas, 2016; Bolaño-García, 2017).

Entre estos materiales también están los Laboratorios Virtuales, que pueden ser utilizados en el aula como complemento de la presentación del tema por parte del docente y de las actividades experimentales de laboratorio, mostrando de una manera interactiva el fenómeno físico que se quiera analizar (Pinto-Salamanca, Sofroni-Esmeral \& Jiménez, 2015; Cáceres \& Amaya, 2016). Además, le permiten al docente acompañar, supervisar y controlar el trabajo de sus estudiantes en tiempo real, mediante la combinación de herramientas de seguimiento dentro del salón de clase (Riveros-Hernández, Nausan-García, García-Miranda \& Palacios-Osma, 2017).

\subsection{Metodología}

Para la elaboración del MEC se utiliza la metodología propuesta por Galvis-Panqueba (1994) la cual comprende cuatro etapas: Análisis, Diseño de la Aplicación, Desarrollo del prototipo y Evaluación.

La población a la que va dirigido el MEC, son los 27 estudiantes de grado undécimo de la Institución Educativa Técnica Valentín García, del municipio de Labranzagrande, Boyacá. Su desarrollo intelectual se considera normal, presentando gran interés por los contenidos novedosos, en especial por aquellos que vienen en formato audiovisual; igualmente, los estudiantes muestran grandes habilidades y destrezas en manejo de la tecnología.

En la etapa de análisis se aplicaron varios instrumentos a la población para recolectar la información necesaria. El primer instrumento es una encuesta con la caracterización sociodemográfica de la población objetivo, luego se aplicó un cuestionario sobre el uso y apropiación de la tecnología, Modelo de Vark, instrumento diseñado para identificar los estilos de aprendizaje y una prueba de conocimiento de 20 preguntas sobre el tema 
de Oscilaciones y Ondas. Esta información sirvió como base para el diseño de la aplicación.

\section{Resultados y discusión}

\subsection{Requerimientos de diseño}

Los resultados obtenidos con la aplicación de los instrumentos de recolección de datos fueron:

La caracterización socio demográfica: 27 estudiantes con edades entre 16 - 19 años, 13 mujeres y 14 hombres, estrato socio-económico 1, pertenecientes a familias disfuncionales, con baja formación intelectual de los padres, 5 tienen internet en casa, 14 tiene computador en casa, en su mayoría provenientes de la zona rural.

La caracterización tecnológica permitió determinar los dispositivos a los que tienen acceso, los cuales son: Smartphone, computadores y tablet; se conectan a internet en estos mismos dispositivos, en un promedio de 2,4 horas diarias. Los usos mayores están en la diversión y las redes sociales; 15 estudiantes no saben que es un MEC y solo 7 han usado MEC en algunas asignaturas; luego de explicarles el concepto, 24 están de acuerdo en utilizar MEC en la clase de física.

El test de conocimiento sobre el tema contiene 20 preguntas de selección múltiple con única respuesta, acompañadas de un enunciado (que presenta una situación, contexto, texto) y cuatro opciones de respuesta, codificadas como A, B, C, y $D$, de las cuales solo una es correcta o válida para la tarea planteada.

El cuestionario evaluó las competencias de uso comprensivo del conocimiento científico, explicación de fenómenos e indagación. En cuanto a la comprensión de la temática de Oscilaciones y Ondas, se obtuvo un promedio de 3,48 respuestas acertadas, con un mínimo de 1 y un máximo de 7 respuestas correctas. Esto permitió establecer que los estudiantes no tenían claridad sobre el concep- to de oscilaciones y ondas, requiriendo un mediador didáctico para lograr la formación científica del conocimiento y su aplicación.

De acuerdo con lo anterior se estableció que la herramienta debe cumplir los siguientes requerimientos:

Población objetivo: El MEC está dirigido a los 27 estudiantes de grado undécimo de la Institución Educativa Técnica Valentín García del Municipio de Labranzagrande.

Área temática: Este MEC pertenece al área de Ciencias Naturales, Eje temático: Entorno Físico, de grado undécimo, a la unidad de Fenómenos Ondulatorios; en el desarrollo de este tema es necesario que los estudiantes logren los siguientes desempeños: definir oscilación y movimiento oscilatorio; reconocer las características principales de una oscilación; definir y clasificar las ondas; caracterizar las ondas y sus propiedades; reconocer las propiedades de las ondas; explicar las consecuencias del movimiento ondulatorio de la materia en el ambiente.

Los anteriores desempeños permitirán a los estudiantes la adquisición de conocimientos, procesos y procedimientos indispensables en el plan de estudios del grado undécimo, así como su aplicación práctica en otras áreas del currículo.

Necesidades Educativas: Con el MEC elaborado se espera aumentar la receptividad, el interés y la asimilación en el logro de los objetivos temáticos, específicamente en el tema de Oscilaciones y Ondas.

El estudio de los estilos de aprendizaje permite conocer mejor a los estudiantes, de tal manera que las actividades desarrolladas en el aula se pueden diseñar basadas en dichas particularidades (Lozano (2006), citado por Rodríguez-Cepeda (2016). Esta metodología toma mayor importancia cuando los estudiantes presentan dificultades en la construcción de los conceptos. 
La aplicación del instrumento VARK permitió establecer que: 9 estudiantes tienen estilo Kinestésico, 7 lector, 6 visual y 5 Auditivo. Es decir, predomina el estilo kinestésico, seguido del Lector, visual y auditivo.

En este sentido, la herramienta a diseñar debe contener una tercera parte de elementos que favorezcan la percepción práctica, real o simulada, que se logra con los simuladores, laboratorios virtuales y cuestionarios. El estilo lector se trabaja con textos explicativos en formato pdf, presentaciones y enlaces a documentos y libros para profundizar en los temas de interés. Los estilos visual y auditivo se trabajan con videos, animaciones e imágenes explicativas.

\subsection{Herramienta propuesta}

Analizados los resultados de los instrumentos aplicados a la población objetivo, se diseñó una herramienta didáctica para desarrollar las competencias en el Área de Ciencias Naturales en la asignatura de Física, teniendo en cuenta la apropiación y uso de la tecnología y los estilos de aprendizaje predominantes. La herramienta es de tipo multimodal, donde se aprecian, en su orden, los aspectos: kinestésico, lector, visual y auditivo, como se ilustra en la figura 1.

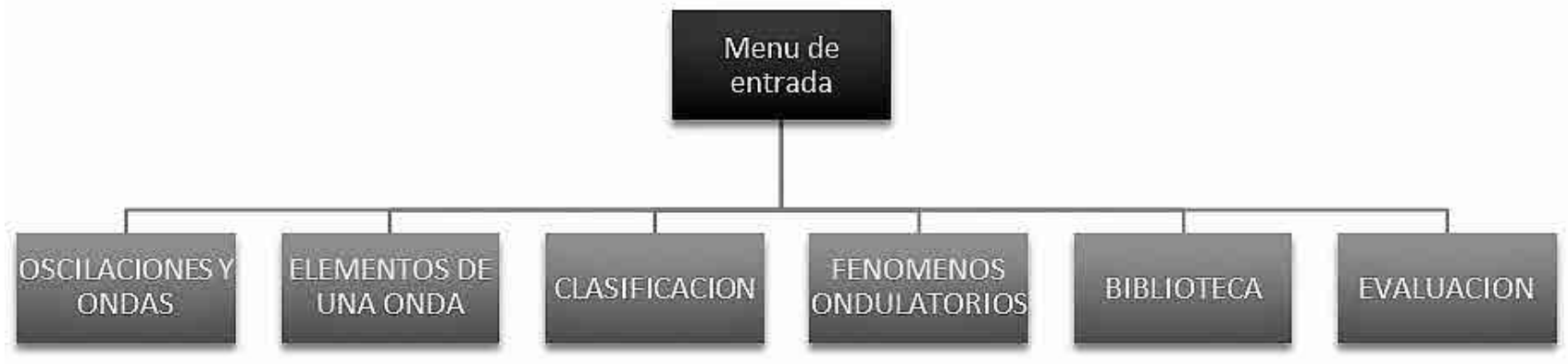

Figura 1. Estructura de la herramienta didáctica: Menú principal.

En la figura 2 se muestra la estructura del Menú secundario del MEC, en el cual se visualizan los contenidos específicos de cada tema para potenciar los estilos de aprendizaje predominantes en cada estudiante. 


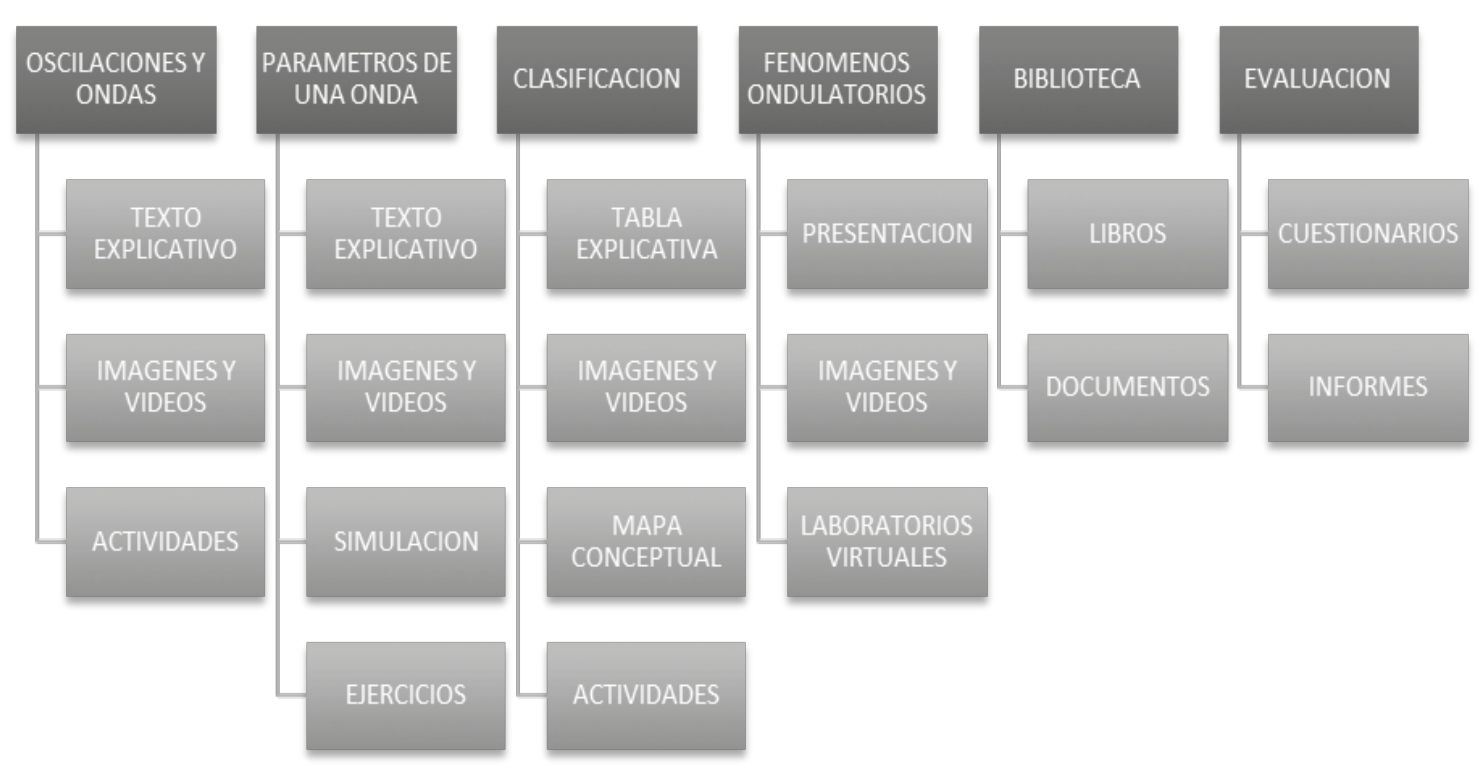

Figura 2. Estructura de la herramienta didáctica MEC: Menú Secundario.

El MEC, de tipo página web para la enseñanza del tema Oscilaciones y Ondas, se elaboró en la plataforma WIX, que permite crear sitios web gratuitos. La ventaja de esta plataforma, además de su gratuidad, es que es compatible con todos los navegadores actuales, dado que las páginas que genera están codificadas en HTML5. Los recursos web utilizados fueron:

- Cuenta gratuita en la plataforma WIX: (www. wix.com).

- Libros de Google Libros: (https://books. google.es/).

- Documentos de Scribd: (https://es.scribd. com/).

- Crucigramas, sopa de letras y cuestionarios: (http://www.educaplay.com/).

Simulador de ondas en una cuerda y laboratorio del péndulo, desarrollado por PhET Interactive Simulations y con licencia de uso y distribución Creative Commons: (http://phet.colorado.edu).
- Videos: (www.youtube.com).

- Imágenes bajo licencia Creative Commons, descargadas de: (https://search. creativecommons.org/).

- URL del producto: (http://edelruiz65.wix.com/ ondas).

Los elementos incluidos en la página son objetos embebidos relacionados con el tema, los cuales están licenciados bajo la licencia Creative Commons. El MEC puede ser consultado en el dispositivo que más usan los estudiantes, en su orden: Smartphone, computadores y Tablet, de acuerdo a la caracterización tecnológica realizada a la población objetivo.

El MEC contiene ocho páginas, a saber: inicio, oscilaciones y ondas, parámetros de una onda, clasificación, fenómenos ondulatorios, biblioteca, evaluación y registro. La página principal, llamada Inicio, es el eje principal del proyecto, pues todas las páginas y subpáginas se conectan con ella, y desde aquí, mediante un menú, se puede acceder a cualquier parte del contenido de la página web, 
ver Figura 3. Las páginas centrales contienen subpáginas que muestran los temas y las diferentes actividades, según sea necesario para la compren- sión de cada lección en la que se divide la temática principal.

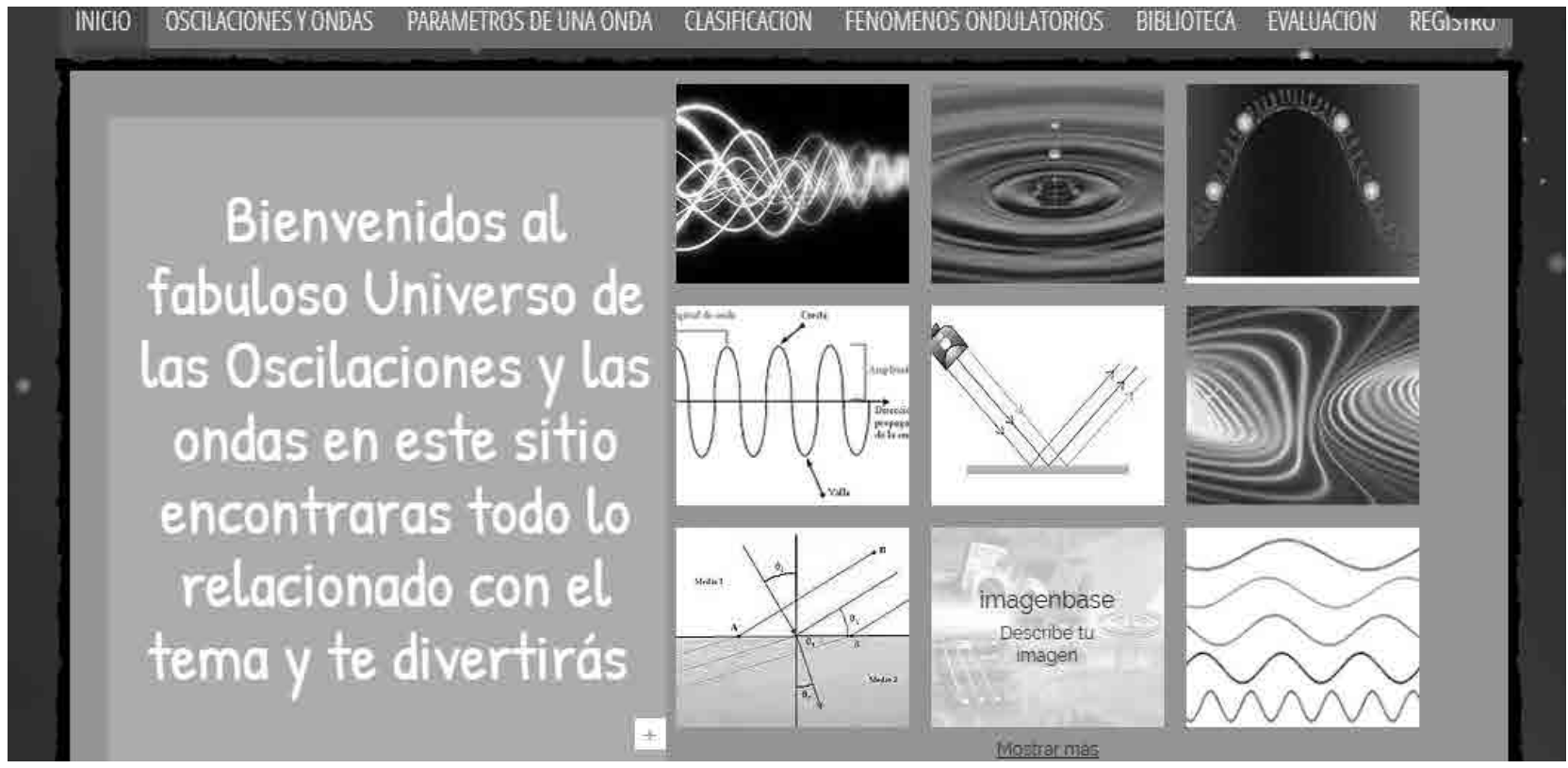

Figura 3. Página de Inicio del MEC.

El principal insumo para el diseño del material fue la caracterización del modo de percepción de los estudiantes; a continuación, se presentan las actividades que favorecen a cada uno de ellos.

Los estudiantes con estilo de aprendizaje kinestésico prefieren lo que involucre la experiencia y la práctica, ya sea simulada o real; se les debe enseñar con ejemplos, ejercicios prácticos y simulaciones. En la Figura 4 se muestra una simulación de parámetros de una oscilación en una cuerda, y la forma de la onda resultante producto de esa oscilación, en la cual se pueden cambiar las condiciones de la situación planteada y observar cómo se modifican los diferentes parámetros. 


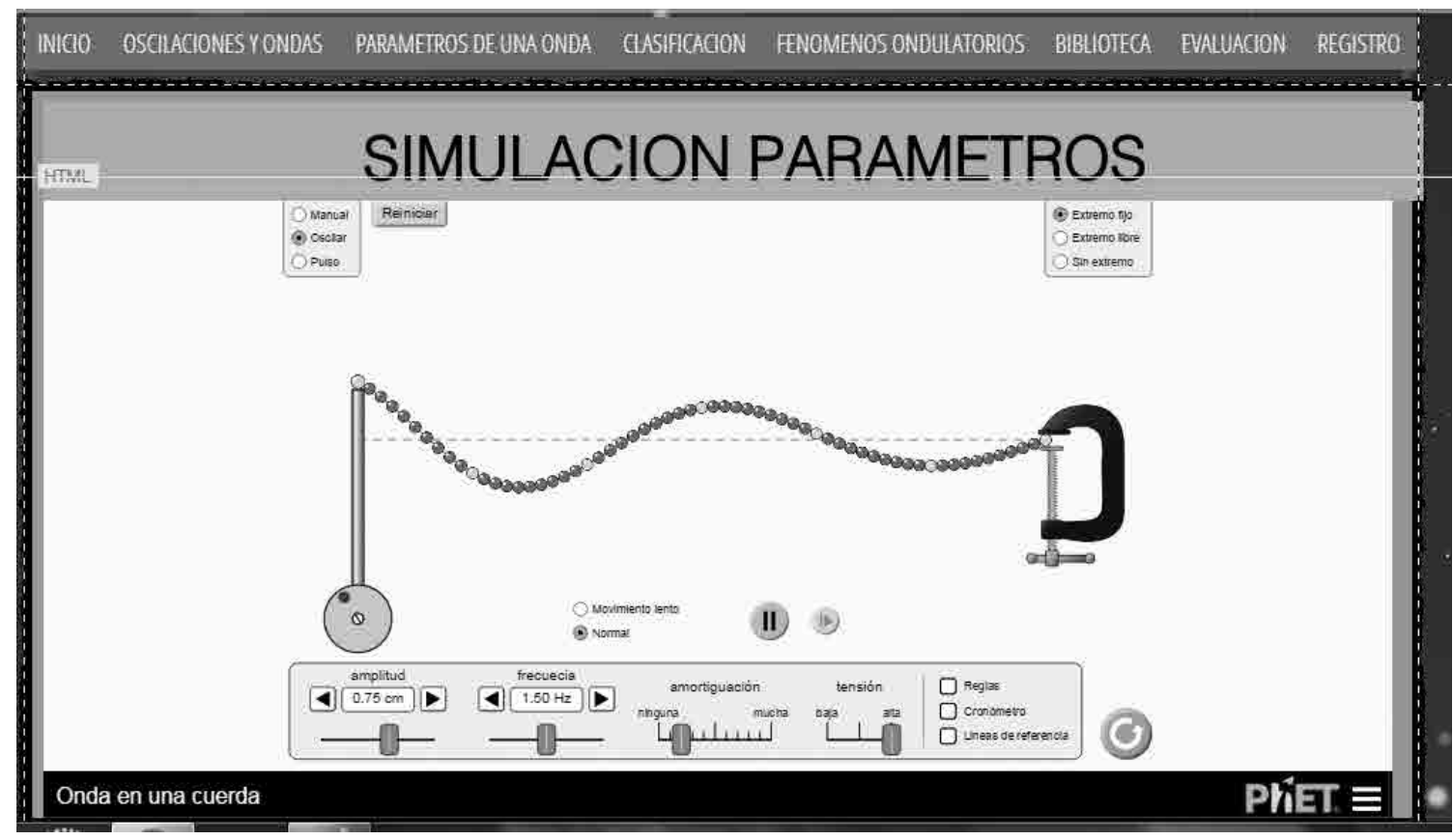

Figura 4. Vista de la subpágina simulaciones.

En la Figura 5 se muestra el laboratorio virtual de la Oscilación de un Péndulo; en esta actividad se entrega al estudiante una guía con las diferentes actividades que debe realizar y el informe a pre- sentar. La aplicación le permite modificar los diferentes parámetros y hacer las mediciones, como si estuviera en un laboratorio real.

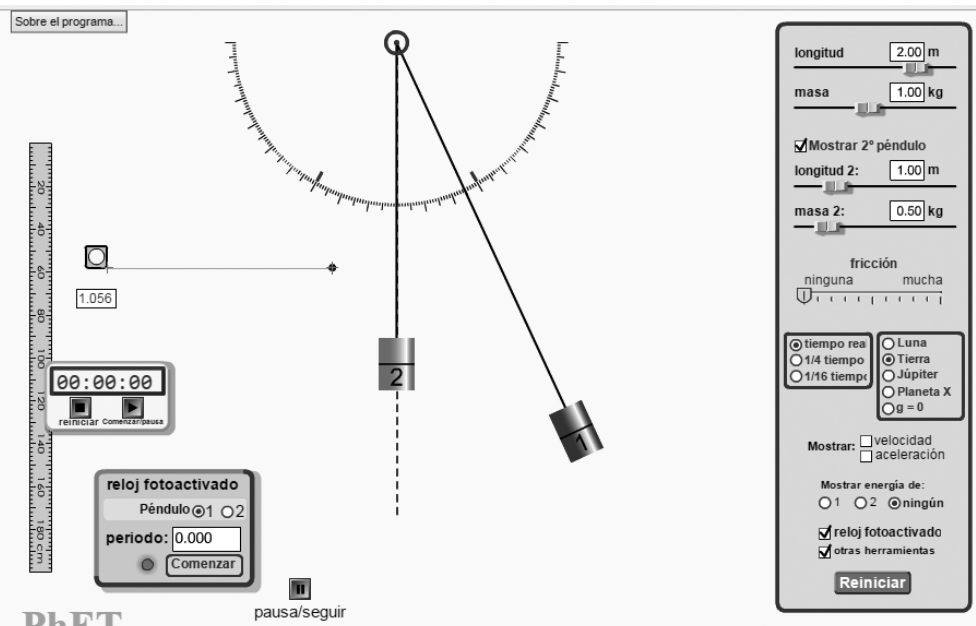

Figura 5. Vista de Laboratorio Virtual de Oscilaciones.

Los estudiantes con estilo de aprendizaje Lector prefieren todo lo relacionado con leer y escribir, revisan material y estudian con notas, diagramas y resúmenes. Para ellos la herramienta cuenta, en 
todos los temas, con una subpágina con texto explicativo y una página principal de biblioteca, con enlaces a artículos y libros para profundizar en los conceptos.

Los estudiantes con estilo auditivo prefieren las exposiciones orales, las conferencias, las discusiones y todo lo que involucre el escuchar. Cuando se evoca el recuerdo, utilizando el sistema de representación auditivo, se hace de manera secuencial y ordenada. Las personas auditivas aprenden mejor cuando reciben las explicaciones oralmente y cuando pueden hablar y explicar esa información a otra persona. Para esta clase de aprendizaje se trabajan los videos explicativos.

Los estudiantes de tipo visual prefieren el uso de imágenes, cuadros, diagramas, círculos, flechas y láminas al momento de estudiar o de aprender conceptos nuevos. Para este estilo de aprendizaje se tienen las galerías de imágenes. En la página de Clasificación se tienen dos subpáginas con un cuadro de clasificación de las ondas y un mapa conceptual, ver Figura 6.

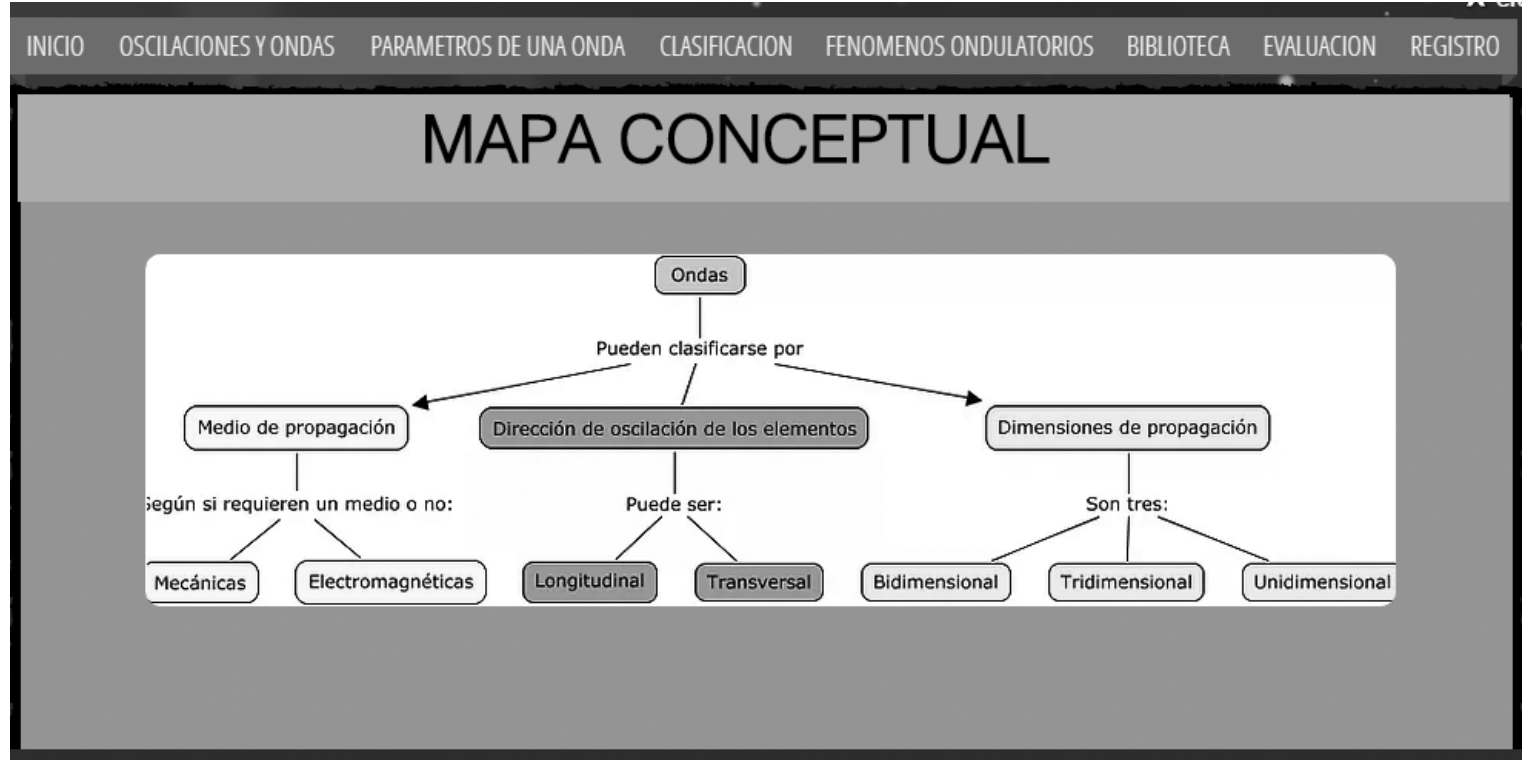

Figura 6. Vista Mapa conceptual subpágina Clasificación.

Las actividades de refuerzo y retroalimentación corresponden a crucigramas, sopas de letras, relaciones y pequeños cuestionarios elaborados en Educaplay. Igualmente, para la evaluación del contenido temático presentado a los estudiantes, en la herramienta didáctica existe una página que contiene cuestionarios tipo Prueba SABER, elaborados en Educaplay.

Este MEC brinda la posibilidad de enseñar a los estudiantes de una manera más dinámica y diverti$\mathrm{da}$, a través de las diferentes actividades que ofrece. En la actualidad la gran mayoría de estudiantes se sienten atraídos por las TIC, siendo preocupa- ción de las instituciones educativas su vinculación a los procesos curriculares (Ricardo et al., 2013; Leguizamón-González, 2011).

En este sentido, materiales como el aquí presentado ofrecen una excelente alternativa didáctica para el manejo de la temática de oscilaciones y ondas. El MEC puede servir como guía para el docente, aprovechando al máximo la motivación producida por los elementos multimedia, a la vez que permite a los estudiantes el acceso a información estructurada favoreciendo el aprendizaje y refuerzo de la temática. 


\section{Conclusiones}

La caracterización de la población objetivo permitió establecer que el estilo de aprendizaje predominante es el kinestésico, seguido por el lector, auditivo y visual. Esta información es vital para seleccionar el material y tipo de actividades que deben ser incluidas en un Material Educativo Computarizado, MEC, dirigido a esta población.

EI MEC diseñado potencializa estos estilos y favorece el aprendizaje significativo y el enfoque cognitivo, en el cual el estudiante es protagonista activo de su formación. La afirmación anterior se fundamenta en el hecho de que los estudiantes asimilan mejor los contenidos, ya que en la herramienta diseñada existen elementos que favorecen su estilo de aprendizaje y las TIC son un factor motivante en el desarrollo del proceso enseñanza aprendizaje.

La principal ventaja de esta herramienta es que hace al estudiante sujeto activo en el proceso de enseñanza - aprendizaje, permitiéndole interactuar a su propio ritmo y puede retroalimentar sus conocimientos, gracias a la variedad de actividades diseñadas de acuerdo a los estilos de aprendizaje.

Con la aplicación de la prueba piloto se observó un alto grado de aceptación del MEC, efectividad, eficiencia y usabilidad por parte de los estudiantes. También se detectaron algunas debilidades tanto en contenido, como tecnológicas y pedagógicas, las cuales se corrigieron para que la herramienta sea funcional y práctica.

\section{Referencias}

Altamirano-Santillán, E., Vallejo-Vallejo, G., \& Cruz-Hurtado, J. (2017). Monitoreo volcánico usando plataformas Arduino y Simulink. Revista de Investigación, Desarrollo e Innovación, 7 (2), 317-329. doi: https://doi.org/10.19053/20278306. v7.n2.2017.6073
Angarita-Velandia, M. A., Fernández-Morales, F. H., \& Duarte, J. E. (2014). La didáctica y su relación con el diseño de ambientes de aprendizaje: una mirada desde la enseñanza de la evolución de la tecnología. Revista de Investigación, Desarrollo e Innovación, 5 (1), 46-55. doi: https://doi. org/10.19053/20278306.3138

Angarita-Velandia, M., Fernández-Morales, F., \& Duarte, J. (2016). Formación de ingenieros interdisciplinarios a través de una metodología activa con temáticas integradoras. Saber, Ciencia Y Libertad, 11 (2), 177-187. doi: http://dx.doi.org/10.22525/ sabcliber2016v11n2.202

Avella-Ibáñez, C. P., Sandoval-Valero, E. M., \& Montañez-Torres, C. (2017). Selección de herramientas web para la creación de actividades de aprendizaje en Cibermutua. Revista de investigación, Desarrollo e Innovación, 8 (1), 107-120. doi: 10.19053/20278306.v8.n1.2017.7372

Bernal-Pinzón, M.L. (2017) ¿quéescriben los niños?, una mirada desde el modelo escuela nueva. Revista de investigación, Desarrollo e innovación, 7 (2), 255-268. doi: https://doi.org/10.19053/20278306. v7.n2.2017.6069

Bolaño-García, M. (2017). Funciones de las herramientas multimedia interactivas para la enseñanza en educación preescolar. Praxis, 13 (1). doi: http://dx.doi.org/10.21676/23897856.2063

Buitrago-Guzmán, S. (2014). Relación entre la convergencia de medios y la experiencia de usuario. Dos iniciativas creativas en Colombia. Revista De Investigación, Desarrollo E Innovación, 4 (2), 79-86. doi: http://dx.doi.org/10.19053/20278306.2958

Cabero-Almenara, J. (2015). Reflexiones educativas sobre las tecnologías de la información y la comunicación (TIC). Tecnología, Ciencia y Educación, 1, 19-27.

Cáceres, C. A., \& Amaya, D. (2016). Desarrollo e interacción de un laboratorio virtual asistido 
y controlado por PLC. Entre Ciencia e Ingeniería, 10 (19), 9-15. Recuperado de: http://www. scielo.org.co/scielo.php?script=sci_arttext\&pi$\mathrm{d}=$ S1909-83672016000100002\&lng=es\&tIng=es.

Cárdenas-Soler, R. N., \& Martínez-Chaparro, D. (2015). El paisaje sonoro, una aproximación teórica desde la semiótica. Revista de Investigación, Desarrollo e Innovación, 5 (2), 129-140. doi: https:// doi.org/10.19053/20278306.3717

Castro-Galeano, J. C., Pinto-Salamanca, M. L., \& Amaya-Quitián, M. F. (2014). Diseño y construcción de una Bobina Tesla de 1680 W, para la enseñanza de conceptos básicos en sistemas eléctricos de potencia. Revista de Investigación, Desarrollo e Innovación, 5 (1), 66-74. Doi: https://doi. org/10.19053/20278306.3142

Cerón-Correa, A., Salazar-Jiménez, A. E., \& Prieto-Ortiz, F. A. (2013). Reconocimiento de rostros y gestos faciales mediante un análisis de relevancia con imágenes 3D. Revista de Investigación, Desarrollo e Innovación, 4 (1), 7-20. doi: $10.19053 / 20278306.2563$

Cortés-Ramos, L. V., Artunduaga, K. J., Suárez, Y. A., \& Marín-Peña, J. A. (2016). Experiencia de aula: modelo del funcionamiento del corazón. Herramienta didáctica del aprendizaje de circuitos eléctricos. Revista Amazonia Investiga, 5 (8), 41-50. Recuperado de: http://www.udla.edu.co/revistas/index. php/amazonia-investiga/article/view/516/pdf_23

De León, I., \& Suárez, J. (2008). El Diseño Instruccional y Tecnologías de la Información y la Comunicación. Posibilidades y Limitaciones. Revista de investigación, (65), 57-81. Recuperado de: http://www. redalyc.org/pdf/3761/376140380003.pdf

Duarte, J., Reyes-Caballero, F., \& Fernández-Morales, F. (2013). La enseñanza de la física en los currículos de ingeniería. Revista de Investigación, Desarrollo e Innovación, 4 (1), 45-55. doi: http://dx.doi. org/10.19053/20278306.2606
Fernández-Morales, F. H., \& Duarte, J. E. (2014). Monitoreo de las variables eléctricas relacionadas con un generador trifásico. Entramado, 10 (2), 314-321. Recuperado de: http://www. scielo.org.co/scielo.php?script=sci_arttext\&pi$\mathrm{d}=$ S1900-38032014000200020\&lng=es\&tIng=es.

Fernández-Morales, F. H., Duarte, J. E., \& Parra-León, L. F. (2014). Herramienta educativa computarizada para el estudio de técnicas ópticas utilizadas en la medida de pequeños desplazamientos. Revista Educación en Ingeniería, 9 (17), 26-35. Recuperado de: https://www.educacioneningenieria.org/index.php/edi/article/viewFile/372/191

Fleming, N. (2001). Encuesta para estilos de aprendizaje vark. Recuperado de: http://www.vark-leam. com/english/index.asp

Galvis-Panqueva, A. (1994). Ingeniería del software educativo. Bogotá, Colombia: Ediciones Uniandes.

García-Amaya, R. A., Fernández-Morales, F. H., \& Duarte, J. E. (2017). Modelo de integración de las TIC en instituciones educativas con características rurales. Revista Espacios, 38 (50), 26. Recuperado de: http://www.revistaespacios.com/ a17v38n50/17385026.html

García-Llorente, H. J. (2015). Multialfabetización en la sociedad del conocimiento: competencias informacionales en el sistema educativo. Revista LasaIlista de Investigación, 12 (2), 225-241. Recuperado de http://www.scielo.org.co/scielo.php?script=sci_arttext\&pid=S1794-44492015000200023\&ln$\mathrm{g}=\mathrm{es} \&$ tlng=es.

Gómez-Soto, J., Sánchez-Toro, Ó., \& Benavides-Salazar, X. (2017). Análisis de patentes como aproximación al diseño conceptual del proceso de obtención de jarabe de lactosuero. Revista de Investigación, Desarrollo e Innovación, 7 (2), 331353. doi: https://doi.org/10.19053/20278306. v7.n2.2017.5453 
González-Calixto, M., Patarroyo-Durán, N., \& Carreño-Bodensiek, C. (2017). El principio de justicia en el aula y la responsabilidad moral del docente, frente a los estilos de aprendizaje. Revista de Investigación, Desarrollo e Innovación, 7 (2), 241253. doi: https://doi.org/10.19053/20278306. v7.n2.2017.4497

González-Otero, A. (2017). El diario: La escritura autobiográfica en su dimensión sociocultural y sus posibilidades cognoscitivas y creativas. LA PALABRA, 0 (30). doi: https://doi.org/10.19053/01218530. n30.2017.6961

Jaramillo, P., Castañeda, P., \& Pimienta, M. (2009). Qué hacer con la tecnología en el aula: inventario de usos de las TIC para aprender y enseñar. Educación y educadores, 12(2).

Jiménez-Pitre, I., Martelo-Gómez, R., \& Peña-Pertuz, M. (2017). Diagnóstico sobre accesibilidad e integración digital dentro del sector universitario colombiano. Saber, Ciencia Y Libertad, 12 (1). Recuperado de: http://www.sabercienciaylibertad.org/ ojs/index.php/scyl/article/view/227

Jiménez-Pitre, I., Vesga, A., \& Martelo, R. (2017). Evaluación de las competencias tecnológicas de los docentes del Instituto Integrado San Bernardo del municipio de Floridablanca, Santander, Colombia. Revista Espacios, 38 (30), 0. Recuperado de: http:// www.revistaespacios.com/a17v38n30/17383001. html

Leguizamón-González, M. C. (2011). Diseño y desarrollo de materiales educativos computarizados (MEC): una posibilidad para integrar la informática con las demás áreas del currículo. Revista Virtual Universidad Católica del Norte, 1(19).

Martínez-Marín, F., \& Cantú-Munguía, I. (2017). Manejo de la simulación en la enseñanza de la ingeniería. Revista Educación en Ingeniería, 12 (24), 5862. doi: http://dx.doi.org/10.26507/rei.v12n24.749
Martínez-Ovalle, S., Reyes-Caballero, F., \& González-Puin, L. X. (2013). Protección radiológica a trabajadores y público en instalaciones que operan radioisótopos industriales. Revista de Investigación, Desarrollo e Innovación, 3 (2), 120-124. doi: $10.19053 / 20278306.2166$

Mercado-Ramos, V. H., Zapata, J., \& Ceballos, Y. F. (2015). Herramientas y buenas prácticas para el aseguramiento de calidad de software con metodologías ágiles. Revista de Investigación, Desarrollo e Innovación, 6 (1), 73-83. doi: https://doi. org/10.19053/20278306.3277

Millán-Rojas, E. E., Gallego-Torres, A. P., \& Chico-Vargas, D. C. (2016). Simulación de una red Grid con máquinas virtuales para crear un entorno de aprendizaje de la computación de alto desempeño. Facultad de Ingeniería, 25(41), 85-92. Recuperado de: http://www.scielo.org.co/scielo.php?script=sci_arttext\&pid=S0121-11292016000100009\&ln$\mathrm{g}=\mathrm{es} \& \mathrm{tlng}=\mathrm{es}$.

Mora-Mendoza, E. Y., Sarmiento-Santos, A., \& Casallas-Caicedo, F. M. (2014). Implementación de un sistema de tratamiento con plasma para gases utilizando una celda de descarga de barrera dieléctrica. Revista de Investigación, Desarrollo e Innovación, 5 (1), 56-65. doi: https://doi. org/10.19053/20278306.3141

Niño-Vega, J. A., Martínez-Díaz, L. Y., \& Fernández-Morales, F. H. (2016). Mano robótica como alternativa para la enseñanza de conceptos de programación en Arduino. Revista Colombiana de Tecnologías de Avanzada, 2 (28), 132-139. doi: $\quad$ https://doi.org/10.24054/16927257.v28. $\mathrm{n} 28.2016 .2476$

Novoa-Ruiz, J. A. (2013). La cuestión integral del siglo XXI. Saber, Ciencia y Libertad, 8 (1), 71-83. Recuperado de: http://www.sabercienciaylibertad. com/ojs/index.php/scyl/article/view/116

Núñez-Pérez, V. (2015). Pedagogía social e interculturalismo: una lectura posible. Revista de Inves- 
tigación, Desarrollo e Innovación, 5 (2), 141-149. doi: 10.19053/20278306.3716

Pabón-Fernández, L., Díaz-Rodríguez, J., \& Pardo-García, A. (2016). Simulación del inversor multinivel de fuente común como variador de frecuencia para motores de inducción. Revista de Investigación, Desarrollo e Innovación, 7 (1), 165180. doi: https://doi.org/10.19053/20278306.v7. $\mathrm{n} 1.2016 .5636$

Parada-Hernández, A., \& Suárez-Aguilar, Z. E. (2014). Influencia de las Tecnologías de la Información y la Comunicación en la apropiación de conceptos de electrónica análoga, en estudiantes de grado séptimo de educación básica. Revista de Investigación, Desarrollo e Innovación, 5 (1), 20-31. doi: https://doi.org/10.19053/20278306.3137

Parra-León, L. F., Duarte, J. E., \& Fernández-Morales, F. H. (2014). Propuesta didáctica para la enseñanza de circuitos eléctricos básicos. Revista de Investigación, Desarrollo e Innovación, 4 (2), 138-147. doi: http://doi.org/10.19053/20278306.2891

Pérez, M. del C., \& Telleria, M. B. (2012). Las tic en la educación: nuevos ambientes de Aprendizaje. Revista de Teoría y Didáctica de las Ciencias Sociales, 18, 83-112.

Pinto-Salamanca, M. L., Sofrony-Esmeral, J. I., \& Jiménez, D. F. (2015). Detección de colisiones con librerías V-Collide y PhysX para interacción virtual con interfaces hápticas. Revista de Investigación, Desarrollo e Innovación, 5 (2), 119-128. doi: $10.19053 / 20278306.3721$

Reinón, P., \& Ramos, J. (2014) Colonialismo digital, atención y lectura en tiempos de cambio. Revista científica de Comunicación y Tecnologías emergentes, 12 (1), 244-266.

Reyes-Caballero, F., Fernández-Morales, F., \& Duarte, J. (2016). Panorama energético. Revista de Investigación, Desarrollo e Innovación, 7 (1), 151-
163. doi: http://dx.doi.org/10.19053/20278306. v7.n1.2016.5605

Ricardo, C., Borjas, M., Velásquez, I., Colmenares, J., \& Serje, A. (2013). Caracterización de la integración de las TIC en los currículos escolares de instituciones educativas en Barranquilla. Zona Próxima, (18), 32-45. Recuperado de: http://www. scielo.org.co/scielo.php?script=sci_arttext\&pid=S2145-94442013000100004\&lng=es\&tlng=es.

Riveros-Hernández, D., Nausan-García, D., García-Miranda, D., \& Palacios-Osma, J. (2017). Desarrollo de un entorno virtual para la simulación de maniobras eléctricas en subestaciones: un caso práctico. Ingeniería solidaria, 13(22). doi: https:// doi.org/10.16925/in.v13i22.1752

Rodríguez-Cepeda, R. (2016). Aprendizaje de conceptos químicos: una visión desde los trabajos prácticos y los estilos de aprendizaje. Revista de Investigación, Desarrollo e Innovación, 7 (1), 63-76. doi: https://doi.org/10.19053/20278306. v7.n1.2016.4403

Salinas, J. (2004). Cambios metodológicos con las TIC. Estrategias didácticas y entornos virtuales de enseñanza-aprendizaje. Bordón, 56 (3-4), 469-481.

Tangarife-Chalarca, D. (2013). Desarrollo de una aplicación web para el montaje de una mesa quirúrgica en el área de traumatología. Revista de Investigación, Desarrollo e Innovación, 4 (1), 32-44. doi: https://doi.org/10.19053/20278306.2124

Velásquez, A. M. V., Ortiz, J. F. Z., \& Rodríguez, A. L. (2016). La relación entre los estilos de aprendizaje y el rendimiento académico en matemáticas en estudiantes de ciclo $v$ de educación secundaria. Journal of Learning Styles, 9 (18). 